\title{
Multiple Resonance Metamaterial Emitter for Deception of Infrared Emission with Enhanced Energy Dissipation
}

Namkyu Lee ${ }^{1, a}$, Boram Yoon ${ }^{1, b}$, Taehwan Kimc, Ji-Yeul Baed ${ }^{\mathrm{d}}$, Joon-Soo Limª Injoong Chang ${ }^{\mathrm{a}}$, Hyung Hee Cho ${ }^{\mathrm{a} *}$

a. Department of Mechanical Engineering, Yonsei University, 50 Yonsei-ro, Seodaemun-gu, Seoul 13722, Korea

b. Korea Aerospace Industries, 78, Gongdanro 1-ro, Sanam-myeon, Sacheon, Gyeongsangnam-do, 52529, Korea

c. Samsung Electronics Inc, Hwaseong, 18448, Korea

d. Agency for Defense Development, Daejeon, 34186, Korea

1. These authors are contributed on paper equally.

* Corresponding author

Tel.: +82 221232828

Fax: +82 23122159

E-mail: hhcho@yonsei.ac.kr 


\section{Spectral emissivity of the multiple resonance metamaterial emitter with the constant property of the polyimide}

Figure S1 shows the spectral emissivity with the constant property of dielectric constant $\left(\varepsilon_{\mathrm{r}}=3.20\right)$ along with the change of the diameter of top gold structure. The dielectric constant is averaged refractive index from 3 to $14 \mu \mathrm{m}$ and square of the averaged refractive index for the dielectric constant. The range of the diameter is from 0.5 to $2.8 \mu \mathrm{m}$ having the step of $0.05 \mu \mathrm{m}$ and the span of the wavelength for the simulation is $0.02 \mu \mathrm{m}$ to capture the resonant behavior as detailed as possible. As shown in Fig. S1, when the constant property of the dielectric constant in a dielectric material, the resonant wavelength is proportional to the diameter of the top gold structure as well known in the metamaterial field ${ }^{[1-3]}$. Except for the effect of the abnormal dispersion caused by the variation of the refractive index, the magnetic resonance occurs at the same resonant wavelength with the multi resonance emitter. Therefore, we arrange the resonant wavelength data in Fig. 3(d).

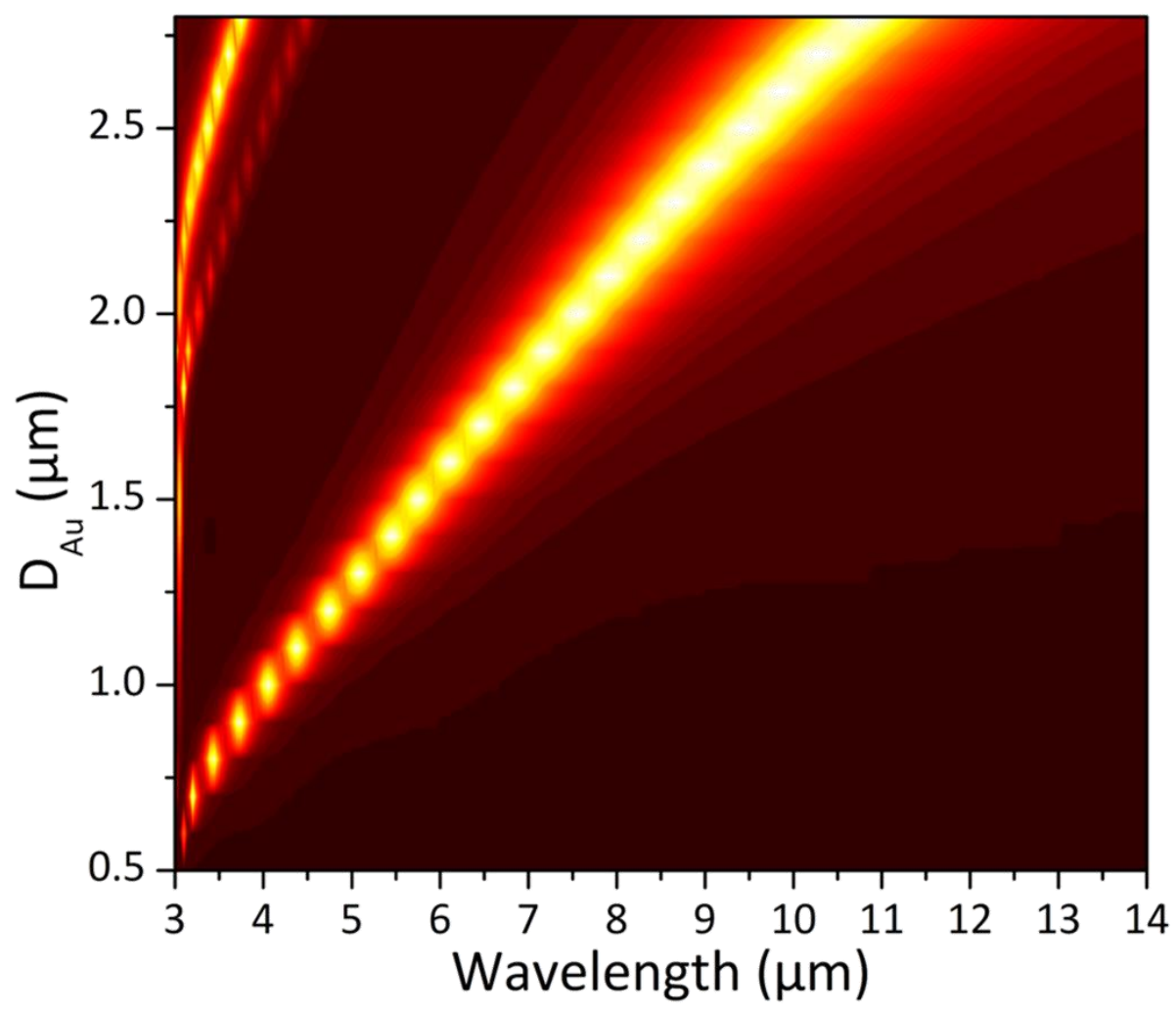

Figure S1. Spectral emissivity by changing the diameter of metal disk at the MDM structure having the dielectric material of constant relative permittivity. 


\section{Details of thermographic measurement}

Figure S2 shows the aluminum bottle coated with the military paint for measuring the thermographic image. The aluminum bottle has a diameter of $150 \mathrm{~mm}$ having the thickness of $2 \mathrm{~mm}$. To measure the boiled water and surface temperature, we use K-type thermocouples with the ice bath as a reference temperature through the triple point of water $\left(0.01^{\circ} \mathrm{C}\right)$. The ambient temperature is $23^{\circ} \mathrm{C}$ by measuring $\mathrm{K}$ type thermocouple. The thermocouples are placed in the air inside the aluminum bottle for measuring the ambient and hot water temperature and attached to the outside surface of the aluminum bottle near the multi-resonance emitter to calibrate the temperature through the IR camera. The calibrated emissivity in IR camera is 0.95 with matching the measured data on the curved surface. The data of thermocouples is gathered by the Agilent (34970a) via software of Benchlink Data Logger.

(a)

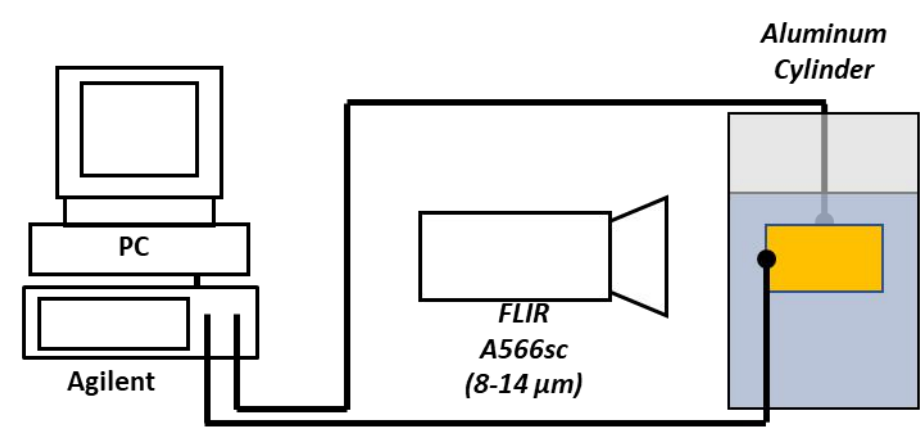

(b)

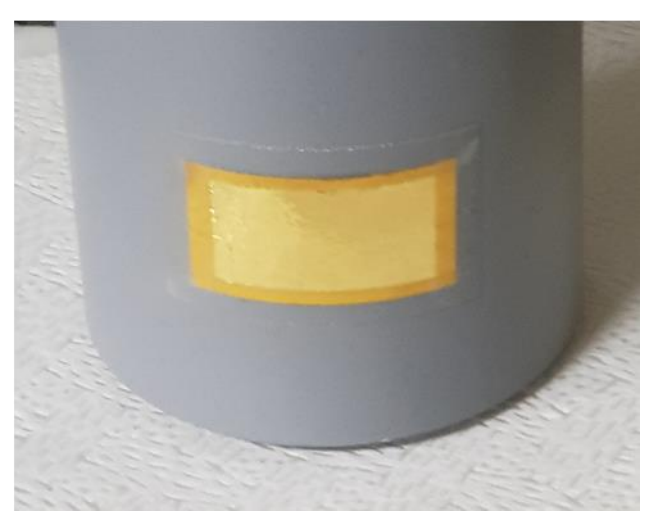

Figure S2. Details of thermographic measurement using IR camera, a) experimental set-up, b) test specimen coated with military paint on the bottle. 


\section{Reference}

1. Landy, N. I.; Sajuyigbe, S.; Mock, J. J.; Smith, D. R.; Padilla, W. J. Perfect Metamaterial Absorber. Phys. Rev. Lett. 2008, 100(20), 207402.

2. Liu, N.; Mesch, M.; Weiss, T.; Hentschel, M.; Giessen, H. Infrared Perfect Absorber and its Application as Plasmonic Sensor. Nano Lett. 2010, 10(7), 2342-2348.

3. Watts, C. M.; Liu, X.; Padilla, W. J. Metamaterial Electromagnetic Wave Absorbers. Adv. Mater. 2012 , 24(23), OP98-120. 